\title{
An Approach to Guidelines Implementation in an E-health System Supporting Chronic Care ${ }^{* * *}$
}

\section{Introduction}

The management of chronic conditions is one of the main challenges for modern health care systems. The results of epidemiological studies indicate that the number of people suffering from chronic diseases increased significantly throughout the last decades. It can reach even $40 \%$ in modern societies. Efficient treatment of patients with chronic diseases requires regular interactions between a patient and health care system. This in turn may result in a considerable rise in health care expenditures.

E-health systems providing remote access to health services are considered as an alternative or support to traditional health care systems, as they allow to decrease costs and simultaneously improve the quality of the life of patients with chronic diseases.

The main goal of the SWOP project (SWOP is the abbreviation of the Polish name meaning The System of Chronic Care Support) is development of an e-health system supporting patients suffering from chronic conditions through self-assessment, telemonitoring and long-term interactions with health care professionals.

The operational concept of the system is shown in Figure 1. On a regular basis patients manually or automatically send results of self-observations or self-measurements specific for their chronic disease. A set of implemented communication modules provide a great flexibility at configuring the parameters, operational modes of sensors and communication channels (WiFi, WAN, GPRS). Entered data are stored in the data base and automatically analyzed to determine-patients' status, trends in disease course and the risk of symptom exacerbation. Then, patients are provided with results of the assessment, which may have a form of messages transmitted from the system to the terminal used by a specific patient, e.g. personal computer or a smartphone (as SMS notifications). The system offers also

* AGH University of Science and Technology, Krakow, Poland

** Jagiellonian University Medical College, Krakow, Poland

*** This work is supported by the National Centre for Research and Development (NCBiR) under Grant No. NR13-0093-10 
capabilities of asynchronous communication between patients and personnel providing support to them (virtual carers, leading physicians or other health professionals, e.g. dietitians or physiotherapist). If needed, an assistance of a specialist physician may be asked. In general, all communication is oriented towards a patient who obtains recommendations dependent on the assessment of his or her status. The system will use of the data provided by all registered users. Moreover, it will offer an option of transferring patient data from an external HL7-compliant health information system (not marked in the figure).

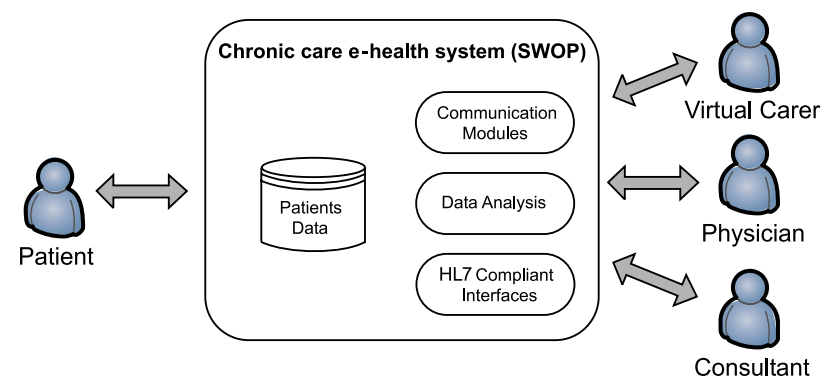

Fig. 1. The operational concept of the SWOP system

The decision support component, which can be considered as a data analysis module, will play a crucial role in the described interaction scenarios. In particular, it will be responsible for assessment of new data entered into the system. This activity constitutes one of the most important steps in the support of self-management performed by the patients within telemonitoring scenarios. The decision support module can be also extended-to other users, involving medical team members or informal carers, if needed.

There are two main aspects of the implementation of decision support in health care systems:

1) The first aspect is related to the source of knowledge used for the development of decision support. Nowadays, the guidelines developed in line with Evidence-Based Medicine (EBM) paradigm usually take the form of narrative recommendations formulated on the basis of available evidence resulting from clinical trials and other types of studies. Typically, guidelines gather knowledge about a single disease, are issued and regularly updated by national or international bodies.

2) The second aspect is associated with the approach to representing and using this knowledge. If expressed with a formal language (FGR - Formal Guidelines Representation) it can be processed and validated by software tools; if linked with a health system components, it may become an executable language interpreted by a computer (CIG Computer Interpretable Guideline).

In this paper we describe an approach to guidelines implementation in the SWOP system. Contrary to various FGR languages developed earlier the decision support will be based on the set of fuzzy rules that on the technical level will be implemented as XQuery 
transformations of XML data. We discuss this approach on an example related to the management of bronchial asthma, as customization of the system to this disease was selected as one of the proof-of-concept exemplifications of the system.

The paper is organized as follows: the next section 2 briefly describes FGRs and provides the discussion, section 3 describes the approach to guidelines implementation within the system, providing also basic information on fuzzy rules to make the presented example more comprehensible. Finally, some concluding remarks are presented in the section 4 .

\section{Discussion and related works}

Various competing Formal Guidelines Represenation (FGR) languages were developed over last ten years. Apart from the oldest, ArdenSyntax [1], which is rule based, almost every one of them, e.g. GLIF [2], SAGE [3], PROforma [4], Asbru [5] or EON [6] attempts to express guidelines in a flowchart like notation. Basic elements of computer interpretable guidelines were summarized in [10]. They include: Eligibility Criteria specifying conditions that must be satisfied by a patient to start a treatment according to a guideline, Goals defining the expected outcome of the guideline, Actions specifying either automatic or manual operations, Decisions corresponding to choices between alternative paths of execution, Classification schemes used at assessments, Patient states (identified illness states) that allow to consider a guideline as a finite state machine leading between subsequent states up to goal and Execution states corresponding to the process context. Most FGRs can employ typical flow control mechanisms found in workflow notations [8] and are capable of starting a subguideline (child process) execution.

There are several factors that limit the application of FGRs for telemonitoring purposes (due to limited capacity we list the most important results of the conducted analysis):

- FGRs were developed keeping in mind that the primary human interaction will undergo between medical professionals and an IT system, whereas in e-health patient plays the central role.

- It is difficult or even undesirable to orchestrate the actions in the system by a centralized process, as the nature of interactions is event-driven.

- Outcomes of the guidelines execution should be context-based. In the scope of the SWOP systems there are contexts related to actors (Patients, Virtual Carers, Physicians and Consultants). A context can be parameterized by the access mode: remote (default) and direct (treated as an option).

- The system should cope with data deviations introduced by patients in subjective selfobservations, sensors of lower quality then used in clinical practice and limited patients skills. This can be achieved by the externalization and adaptation of guidelines parameters.

- Several constructs appearing in FGRs are superfluous in reference to the system goals and too costly to implement. 
The listed above drivers led us to the decision to represent executable guidelines within the SWOP system as a set of fuzzy rules. Such an approach satisfies all requirements elicited during the analysis: it is much simpler than process-oriented FGRs, allows adaptation at the level of fuzzy membership functions and it is well aligned with the nature of guidelines, which reports the evidence and recommends decisions and assessments based mainly on statistical trials.

There are a few publications on how guidelines were represented in telemonitoring systems. Usually they employ rules [9] or decision trees [10]. The fuzzy based approach seems to be rare in this particular field, although there is a large literature on the application of various forms of fuzzy reasoning as a support for diagnosis and making decisions related to therapy plans, e.g. $[11,12,13]$. Such a large interest is due to the nature of the medical knowledge collected in guidelines (based on statistically interpreted trials) and the language used to express it (see discussion on decidability and executability in [13]).

\section{Approach to guidelines implementation in the SWOP system}

In this section we describe the approach to guidelines implementation within SWOP system. This approach is consistent within five goals that were stated by medical professionals involved in the project:

1) Assessment of the disease state based on available information (including current values of monitoring parameters, subjective symptoms, historical data, and care plan).

2) Assessment of the possibility of exacerbation occurrence.

3) Informing a patient (or patient's family) about disease state, including alerting in case of exacerbations. Informing is not visible in traditional guidelines representations, as they assume direct interaction between medical staff and with patient, however, it is an important issue in telemonitoring systems.

4) Recommendations concerning educational materials related to the patient's disease.

5) Optionally, recommendations concerning changes in the care plan (including changes of medication, their dosage and additional examinations). They are visible only for medical staff. Patients receive information about suggested visit to the physicians office.

\subsection{Decision module}

The decision module is responsible for processing the set of rules formalizing the guidelines. The course of its operation is as follows:

1) Patient records stored in the data base are loaded into the context variables.

2) The decision module executes rules for which the input variables are defined in the order specified by their priority (first more salient rules); the rules may produce results that are input for other rules (Fig. 2).

3) Output variables are processed with built in software functions that store values in the database, produce events or send notifications. 


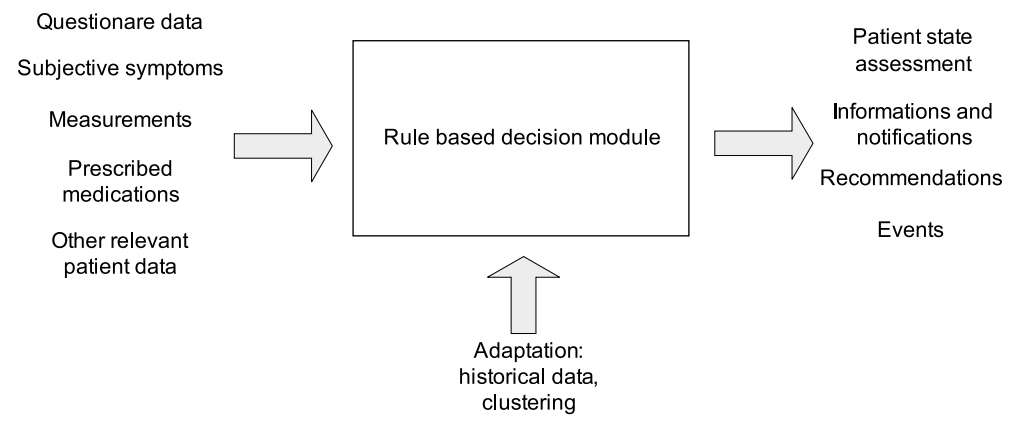

Fig. 2. Inputs and outputs of the decision module

All variables and symbolic values appearing as premises or output values (including actions) are described and classified in the ontology. The consistency of rules with regard to the vocabulary defined in the ontology is checked while editing them and during deployment.

\subsection{Controlling the guideline execution}

In the approach adopted for the SWOP platform assume that the inferences made by the decision module are triggered by events. Events carry information about

- the changes to which the system should respond (e.g. arrival of new patient data or human operator decisions),

- the execution context indicating the access method (remote or direct) and the involved agent: automated system, patient, physician, etc.

This aforementioned information allows to select the proper, context-based set of rules which should be used to process an event. To provide greater flexibility, the configuration of the inference engine (i.e. the mapping between contexts and rule sets) is a part of the system ontology defining various types of contexts and classifying rules.

Although implementing guidelines in the SWOP system we have adopted a solution being to some extend in opposition to the process centric approach, the platform is capable of executing complex processes using the event feedback mechanism (Fig. 3).

The decision module is triggered by events stored in its input queue. An activation of the module can be seen as an occurrence of an activity (task) within a certain non-explicitly specified process. Such a process can consist of a single activity corresponding to one execution of a rule set producing output data, sending notifications, etc. However, some rules can be configured to generate events that are send back to the input queue. This allows to define casual relationships between tasks that combined together build up a process occurrence. From this standpoint, rule sets can be treated as stateless activities, whereas events stored in the input queue as stored task states. 


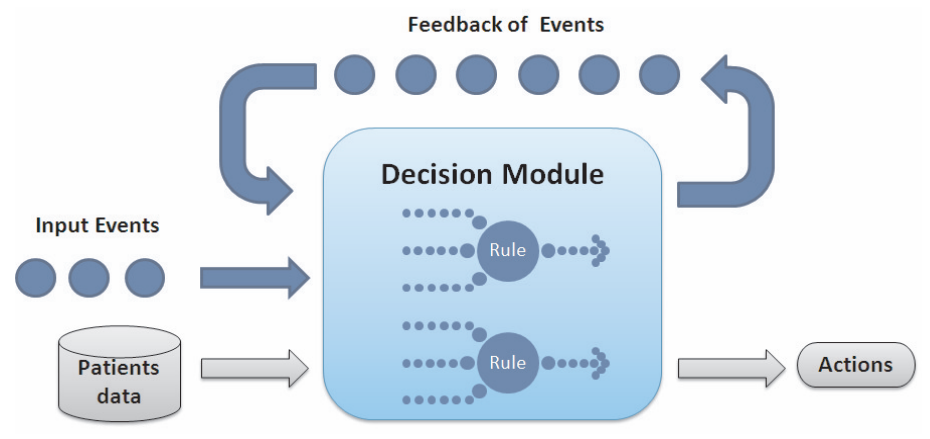

Fig. 3. The decision module is activated by event occurrences

\subsection{Fuzzy rules and fuzzy reasoning}

Fuzzy rules are proven to be an efficient decision support mechanism in many areas. [14] control, home appliances, cameras, embedded systems, etc. They enable implementing even a very sophisticated control mechanism with the use of small sets of rules. Rules in the form proposed by Mamdani are simple conditional instructions:

$$
\text { IF } v a r_{1}=\text { value }_{11} \text { AND var }{ }_{2}=\text { value }_{21} \ldots \text { THEN var }{ }_{\text {out }}=\text { out }
$$

The terms var ${ }_{\mathrm{x}}$ appearing in rules are so called linguistic variables, and value ${ }_{\mathrm{x}}$ and out are fuzzy sets.

Fuzzy sets are described by a membership function defining the confidence factor belonging to the interval $[0,1]$ that a particular element is a member of the set. This concept is explained in the Figure 4 defining two fuzzy sets pev_low and pev_normal representing classes of PEV (peak expiratory volume) parameter values. The membership function for pev_low fuzzy set asserts that all values below $70 \%$ belong surely to the set. Similarly, all values above $90 \%$ belong to the pev_normal fuzzy set with confidence 1.0. The values between $70 \%$ and $90 \%$ belongs to both sets, however their membership vary from 0 to 1.0 .

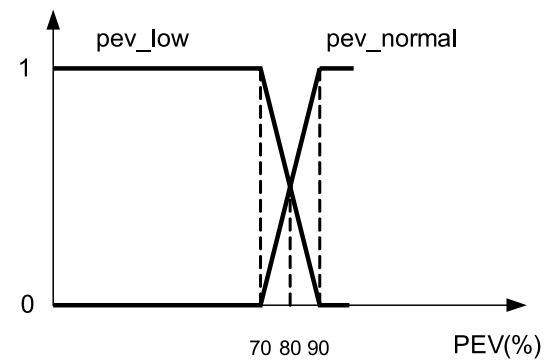

Fig. 4. The standard form of the membership function for the PEV parameter determined based on GINA 2010 guidelines 
The general outline of a decision module based on the fuzzy rule sets is presented in the Figure 5. The following phases in its operation can be distinguished:

- Fuzzification: values of the parameters are mapped to the fuzzy sets according to defined membership functions. Then, fuzzy sets are assigned to linguistic variables. It should be noted, that a variable can be bound to multiple values. E.g., assuming the membership function in Figure 4, for the PEV value equal to $80 \%$ a linguistic variable related to the parameter will be assigned with two values: (pev_low, 0.5) and (pev_normal, 0.5).

- Inference: consisting in applying defined rules and assigning values to output variables (the results of multiple rules execution are stored in output linguistic variables). Before further processing the contents of the output variables is summed using different metrics, typically based on maximal on average membership value.

- The last step consists in defuzzification, i.e. converting fuzzy values to crisp (actual output). Defuzzification is particularly important in control applications that should produce analogue output. In the scope of the SWOP it is rather expected to obtain a single discrete value on the output of the decision module, e,g, assessment of the patient state or a list of discrete values, as possible decisions with assigned priority based on the membership values.

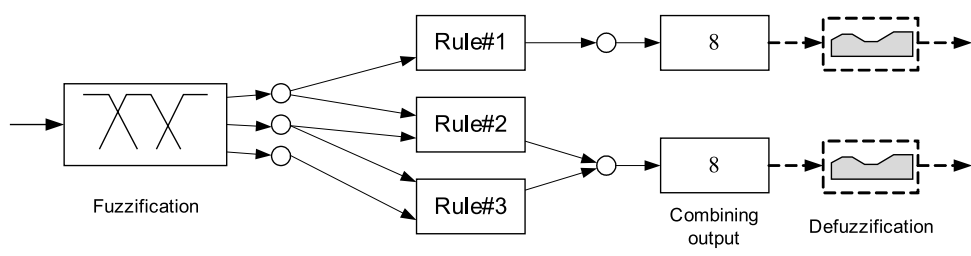

Fig. 5. Stages of the inference with the use of fuzzy rules

\subsection{Example of rule-based a guideline model}

In this section we present a partial example of a guideline model adopting the fuzzy rules approach. Figures 6 and 7 present rules aimed at assessment of the asthma control according to GINA 2010 recommendations [15]. The set of rules can be interpreted as colored Petri nets [16], where transitions depicted as rounded rectangles correspond to applying membership functions and transitions shown as rectangles with sharp corners represent rules. The value in the parenthesis following the rule name denotes its priority.

The course of inference is conceptually depicted in Figure 8. Input variables: PEV equal to $72 \%$ and number of daytime activity limitations equal to 4 are converted in the fuzzification process into values of linguistic variables:

pev $=\{($ pev_normal, 0.1), $($ pev_low, 0.9) $\}$ and dal $=\{($ high, 1.0$)\}$.

Then, applying rules R2 and R6 the value of the linguistic variable is calculated:

asthma_control $=\{$ (partially_controlled, 0.9), (partially_controlled,1.0) $\}$. 


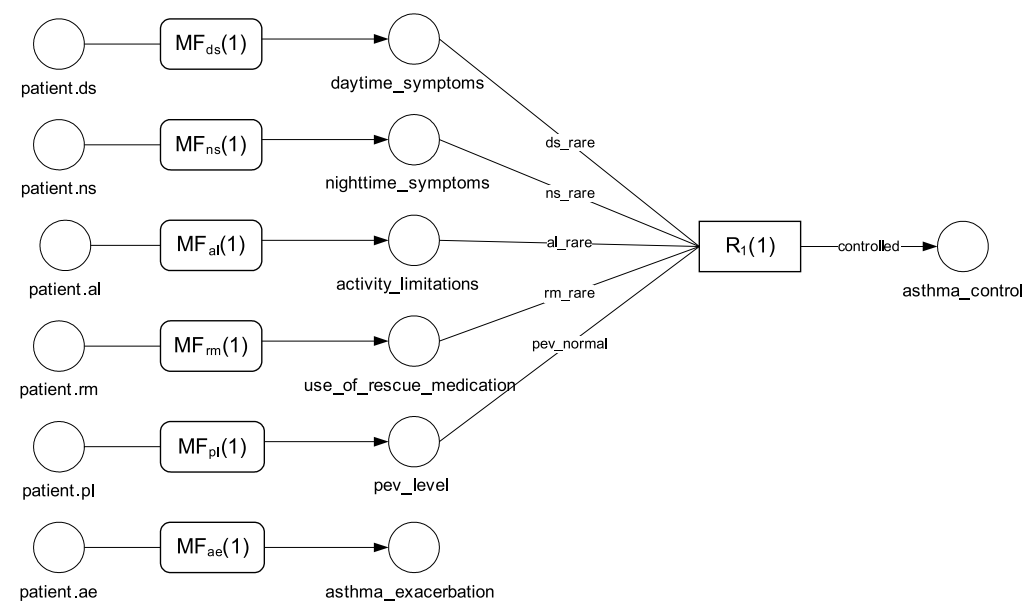

Fig. 6. The first set of rules determining the asthma control

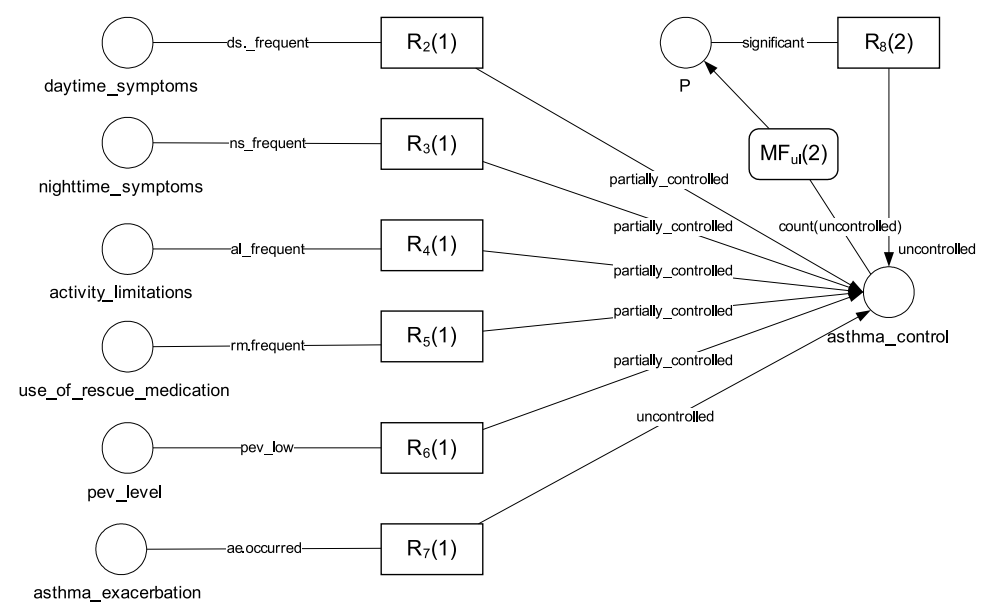

Fig. 7. Asthma control rules (the rules deciding about partially controlled or uncontrolled asthma are shown)

In fact, the asthma_control variable contains another member (controlled, 0.1 ) resulting from $\mathrm{R}_{1}$ execution. As the membership factor has rather a residual value, we decided not to show it on subsequent figures to maintain clarity.

When combining the asthma_control values, according to the membership values it is finally decided, that the disease is in the state of partial control. The obtained result is further processed by other rules defining required actions (e.g. notifications and recommendations). 


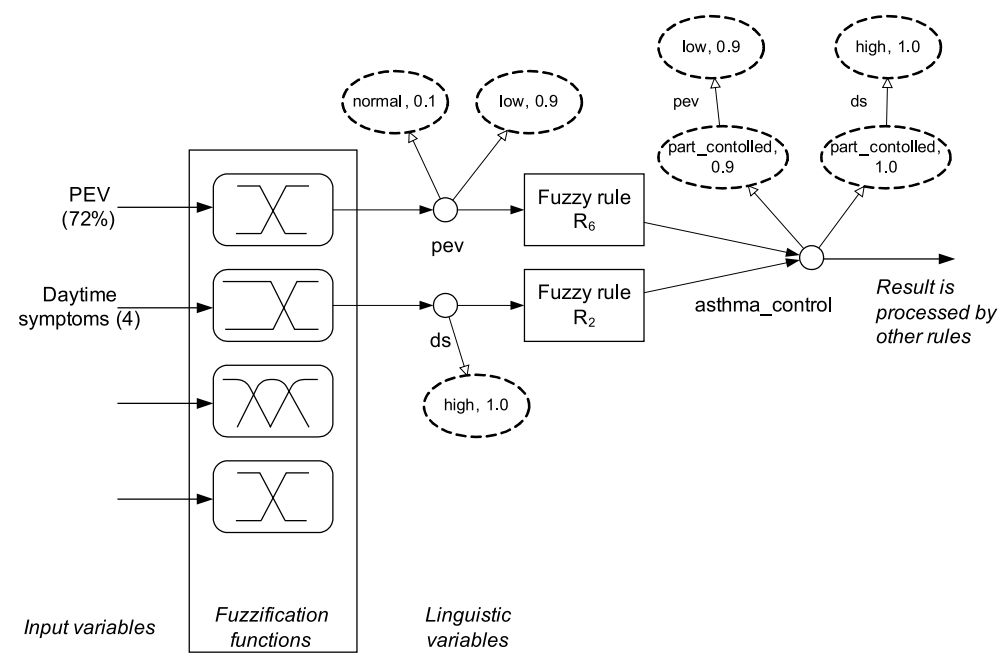

Fig. 8. The course of the inference process

\subsection{Implementation of the reasoning engine}

The reasoning engine is not fully implemented yet. A decision has been made to base it on XML technologies:

- Linguistic variables and fuzzy sets are represented by XML nodes.

- Rules (defined also in XML in the user-friendly interface) are translated into XQuery [17] statements.

- It was assumed, that as far as possible the results of inference should preserve the inference trace to provide evidence for decisions.

The values of linguistic variables pev and $d s$ referred in Figure 8 are represented by XMLnodes shown in Figure 9. After the rules execution the asthma_control variable is assigned with an XML document defining the values with membership coefficients, as well as premises, which were used to calculate them (Fig. 10).

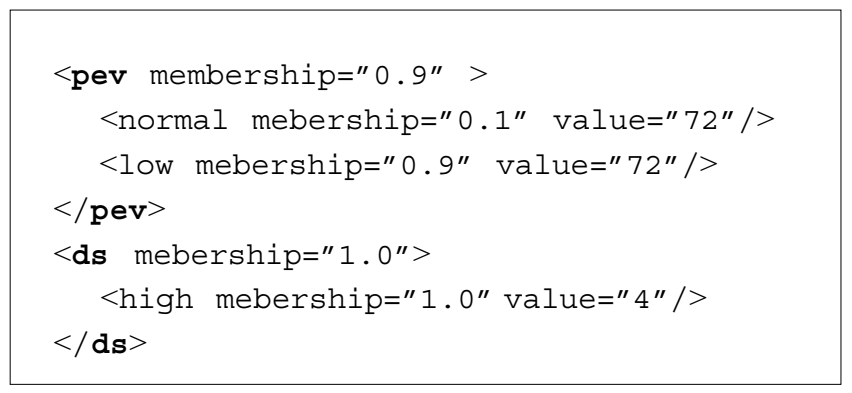

Fig. 9. Values of the linguistic variables pev and $d s$ 


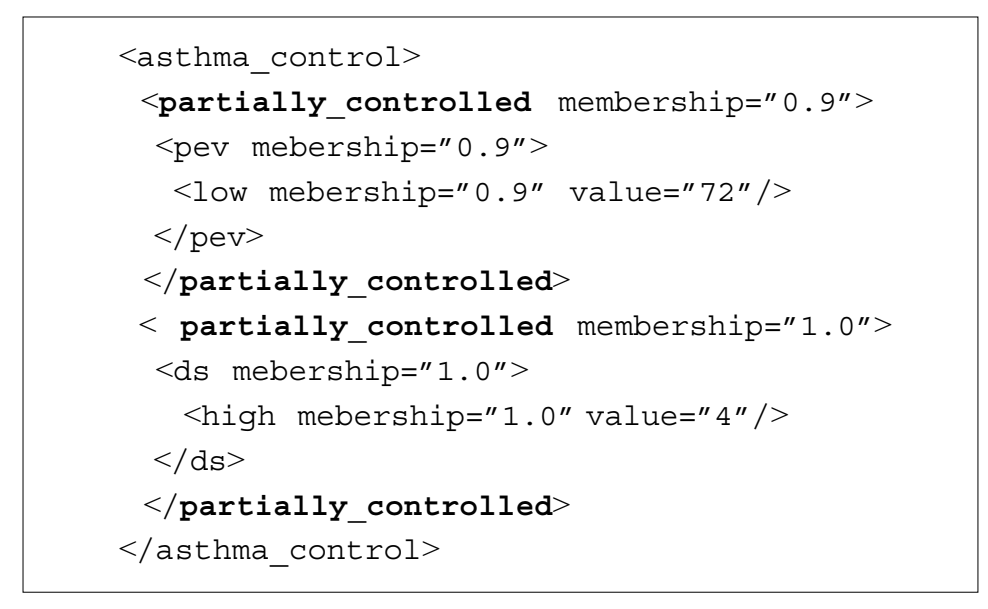

Fig. 10. An XML document representing the set of values for the variable asthma_control obtained by activation of two rules $\mathrm{R}_{6}$ and $\mathrm{R}_{2}$

At present the works focus on automatic translation of rules into XQuery statements. In the future it is planned to integrate it with a tool supporting XQPN (XQuery Petri Networks) - colored Petri networks for manipulation of XML documents [18].

\subsection{Adaptation of a guideline}

We assumed that a set of defined fuzzy rules applies to all patients (it should be remarked that it is context based). The adaptation goals are realized at the level of membership functions. Such adaptation can be:

- individual, i.e. related to a particular patient,

- related to a group based on a common characteristic, e.g. patients using common hardware, exposed to similar environment factors or belonging to a particular age range.

Figure 11 shows an example of a modified membership function for the PEV monitoring parameter that can be obtained as the result of adaptation.

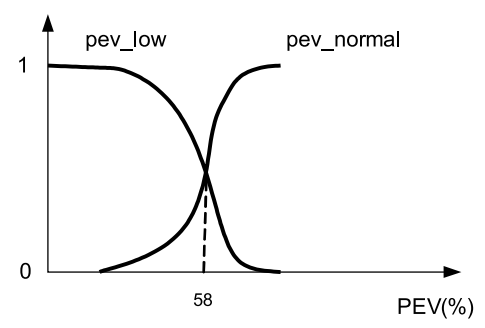

Fig. 11. Example of modified membership function obtained from an adaptation process 


\subsection{Guideline representation}

The guideline is formalized in the XML language and consists of two basic elements:

- Rule sets: each rule set is attributed with the context specifying the concerned agent and the access mode. Each rule in the set has a priority and corresponds to the equation (1). As it was observed, that a rule system that can be elicited from medical guidelines very often can be incomplete, it is obligatory to define default rules returning "safe" outputs with a very low membership factor.

- Specifications of standard membership functions (coordinates of the polyline points. These functions are used by default, however they can be changed for a particular patient.

The rule editor is capable of editing both parts of the specification. Moreover, it uses a vocabulary defined in ontologies. specifying: input data, output data and actions, events, linguistic variables and fuzzy sets appearing in the definitions of rules, agents and rule execution contexts. During the specification of rules these elements are selected from lists fed with appropriate names of ontology concepts.

This specification will be translated into executable representation in the form of XQuery statements, however, other development directions, i.e. reasoning at the OWL level with use of automatically generated SWRL rules and Pellet reasoner are envisaged as an alternative approach.

\section{Conclusions}

In this paper we present an approach to guideline representations adopted in the SWOP e-health system. The decision to represent them in the form of fuzzy rules was preceded by the analysis of formal guidelines representation languages, e.g. GLIF [2], PROforma [4] and others and their limitation with respect to constraints and drivers governing telemonitoring systems. Long lasting discussions between the medical professionals and representatives of the IT world indicated, that there is a substantial gap between how guidelines are perceived by both parties. IT people expect, that a knowledge collected in guidelines can be easy transformed into algorithms or strict rules, whereas the other party emphasizes the fact that guideline recommendations are often very general, present several alternatives, and dependent on many factors. An approach based on fuzzy rules seems to be a rational compromise in this area. Moreover, it provides a framework allowing for personalized adaptation that can be done independently based on the statistical analysis of the collected data without changes into the structure of the rules.

This discussion of the presented example aimed to show, that rules can be easily expressed with a colored Petri nets language, and in particular for the XML based solution with XQPN nets [18]. It is planned to perform validation of the rules defined by medical professionals by simulations with use of the tool supporting XQPN. 


\section{References}

[1] Jenders R.A., Corman R., Dasgupta B., Making the standard more standard: a data and query model for knowledge representation in the Arden syntax. AMIA Annu Symp Proc., 2003, pp. 323-30.

[2] Boxwala A.A., Peleg M., Tu S., Ogunyemi O., Zeng Q.T., Wang D., Patel V.L., Greenes R.A., Shortliffe E.H., GLIF3: a representation format for sharable computer-interpretable clinical practice guidelines. J Biomed Inform 37(3), 2004, pp. 147-161.

[3] Tu S.W., Campbell J.R., Glasgow J. et al., The SAGE Guideline Model: Achievements and Overview. J Am Med Inform Assoc. September-October, 14(5), 2007, pp. 589-598.

[4] Sutton D.R., Fox J., The Syntax and Semantics of the PROforma guideline modelling language. J Am Med Inform Assoc. Sep-Oct, 10(5), pp. 433-43.

[5] Shahar Y., Miksch S., Johnson P., The Asgaard project: A task-specific framework for the application and critiquing of time-oriented clinical guidelines. Artificial Intelligence in Medicine (14), 1998, pp. 29-51.

[6] Tu S.W., Musen M.A., Modeling Data and Knowledge in the EON Guideline Architecture. Proc. MedInfo 2001, London, UK, 2001, pp. 280-284.

[7] Sonnenberg F.A., Hagerty C.G., Computer-interpretable clinical practice guidelines. Where are we and where are we going? Yearb Med Inform, 2006, pp. 145-158.

[8] Mulyar N., van der Aalst W.M.P., Peleg M., Research Paper: A Pattern-based Analysis of Clinical Computer-interpretable Guideline Modeling Languages. JAMIA 14(6), 2007, pp. 781-787.

[9] Finkelstein J., Friedman R.H., Telemedicine system to support asthma self-management. Information Technology Applications in Biomedicine, 2000, pp. 164-167.

[10] Mohktar M.S., Basilakis J., Redmond S.J., Lovell N.H., A guideline-based decision support system for generating referral recommendations from routinely recorded home telehealth measurement data. Engineering in Medicine and Biology Society (EMBC), 2010, pp. 6166-6169.

[11] Steimann F., On the use and usefulness of fuzzy sets in medical AI. Artificial Intelligence in Medicine, vol. 21, Issues 1-3, January-March, 2001, pp. 131-137.

[12] Bates J.H., Young M.P., Applying fuzzy logic to medical decision making in the intensive care unit. Am J Respir Crit Care Med, 167, 2003, pp. 948-952.

[13] Liu J.C.S., Shiffinan R.N., Operationalization of Clinical Practice Guidelines Using Fuzzy Logic. Proc AMIA Annu Fall Symp., 1997, pp. 283-287.

[14] Ross T.J., Fuzzy Logic with Engineering Applications. Third Edition, John Wiley \& Sons, 2010.

[15] GINA - the Global Initiative for Asthma. http://www.ginasthma.org/guidelines-gina-report-global-strategy-for-asthma.html.

[16] Jensen K., Coloured Petri Nets. Basic Concepts. Analysis Methods and Practical Use, vol. I-III, Springer Verlag, 1995/96.

[17] XQuery 1.0: An XML Query Language (Second Edition), http://www.w3.org/TR/xquery/.

[18] Szwed P., XQPN - colored Petri nets for processing XML data with XQuery language. Przegląd Elektrotechniczny (Electrical Review), R. 86, No. 9, 2010, pp. 221-225. 